\title{
Impact of Impurities on the Electrical Conduction of Anisotropic 2D Materials
}

Sun, Jianbo; Passacantando, Maurizio; Palummo, Maurizia; Nardone, Michele ; Kaasbjerg, Kristen; Grillo, Alessandro ; Di Bartolomeo, Antonio ; Caridad, Jose; Camilli, Luca

Published in:

Physical Review Applied

Link to article, DOI:

10.1103/PhysRevApplied.13.044063

Publication date:

2020

Document Version

Publisher's PDF, also known as Version of record

Link back to DTU Orbit

Citation (APA):

Sun, J., Passacantando, M., Palummo, M., Nardone, M., Kaasbjerg, K., Grillo, A., Di Bartolomeo, A., Caridad, J., \& Camilli, L. (2020). Impact of Impurities on the Electrical Conduction of Anisotropic 2D Materials. Physical Review Applied, 13(4), [044063 ]. https://doi.org/10.1103/PhysRevApplied.13.044063

\section{General rights}

Copyright and moral rights for the publications made accessible in the public portal are retained by the authors and/or other copyright owners and it is a condition of accessing publications that users recognise and abide by the legal requirements associated with these rights.

- Users may download and print one copy of any publication from the public portal for the purpose of private study or research.

- You may not further distribute the material or use it for any profit-making activity or commercial gain

- You may freely distribute the URL identifying the publication in the public portal 


\title{
Impact of Impurities on the Electrical Conduction of Anisotropic Two-Dimensional Materials
}

\author{
Jianbo Sun $\odot,{ }^{1}$ Maurizio Passacantando $\odot,{ }^{2}$ Maurizia Palummo, ${ }^{3,4}$ Michele Nardone, ${ }^{2}$ \\ Kristen Kaasbjerg, ${ }^{1}$ Alessandro Grillo, ${ }^{5}$ Antonio Di Bartolomeo $\odot,{ }^{5}$ José M. Caridad, ${ }^{1,}{ }^{*}$ and \\ Luca Camilli® ${ }^{1,4, \dagger}$ \\ ${ }^{1}$ Department of Physics, Technical University of Denmark, Ørsteds Plads, 2800, Kgs. Lyngby, Denmark \\ ${ }^{2}$ Dipartimento di Scienze Fisiche e Chimiche, Università di L'Aquila, via Vetoio, Coppito, 67100 L'Aquila, Italy \\ ${ }^{3}$ INFN, Section at Roma "Tor Vergata", via della Ricerca Scientifica 1, 00133 Roma, Italy \\ ${ }^{4}$ Dipartimento di Fisica, Università degli studi di Roma "Tor Vergata", via della Ricerca Scientifica 1, 00133 \\ Roma, Italy \\ ${ }^{5}$ Physics Department "E. R. Caianiello", University of Salerno and CNR-SPIN Salerno, via Giovanni Paolo II n. \\ 132, Fisciano 84084, Italy
}

(Received 3 December 2019; revised manuscript received 17 February 2020; accepted 7 April 2020; published 24 April 2020)

\begin{abstract}
Anisotropic two-dimensional materials possess intrinsic angle-dependent physical properties that originate from their low crystal symmetry. Yet, how these properties are affected by external impurities or structural defects in the material is still wholly unclear. Here, we address this question by investigating the electrical transport in the anisotropic layered model system germanium arsenide. First, we show that the ratio of conductivities along the armchair and zigzag crystallographic directions exhibits an intriguing dependence with respect to both temperature and carrier density. Then, by using a conceptually simple model, we demonstrate that this unexpected behavior is directly related to the presence of impurityinduced localized states in the band gap that introduce isotropic hopping conduction. The presence of this conduction mechanism in addition to the intrinsic band conduction significantly influences the anisotropic electrical properties of the material, especially at room temperature, i.e., at application-relevant conditions.
\end{abstract}

DOI: 10.1103/PhysRevApplied.13.044063

\section{INTRODUCTION}

Anisotropic two-dimensional (2D) materials represent a class of materials with low crystal symmetry and strong anisotropic physical properties [1]. They have recently attracted a great deal of interest as they hold promise for the realization of alternative angle-dependent devices, such as digital inverters [2,3], polarization-sensitive photodetectors [4,5], and thermoelectric devices [1]. In addition to the largely studied black phosphorus [6], a vast number of anisotropic 2D materials have been explored recently, including for instance group-IV monochalcogenide (e.g., GeS [7]), low-symmetry transition-metal dichalcogenides (e.g., $\mathrm{ReS}_{2}$ [8]), low-symmetry metal chalcogenide(s) (e.g., GaTe [9]), and the most recently discovered group-IV-V compounds (e.g., GeAs [10,11], GeP [12]). Due to their ultrathin nature, the physical properties of anisotropic 2D materials are expected to be highly sensitive to inevitable structural defects and extrinsic charged impurities [13] as well as to many other factors,

\footnotetext{
*jcar@dtu.dk

†luca.camilli@roma2.infn.it
}

e.g., substrates, environment, and surface roughness [14]. In particular, it is reasonable to believe that these extrinsic elements will significantly alter the intrinsic electrical anisotropic conduction in these materials. Such information is, of course, crucial for exploiting anisotropic 2D materials in practical applications, and yet our understanding of this topic is currently inadequate [6,15-17]. Filling this knowledge gap is the objective of this work. To this aim, we fabricate field-effect devices made from the model anisotropic layered system GeAs. GeAs exhibits a high anisotropy ratio [10,11] (more than 3 times larger than black phosphorous) and a band gap relevant for optoelectronic applications (approximately $0.5 \mathrm{eV}$ for bulk and approximately $2 \mathrm{eV}$ for monolayer) [18-21]. We show that below $180 \mathrm{~K}$ the electron transport is mainly due to variable range hopping (VRH) via localized states in the band gap created by impurities, while band conduction in the material plays a dominant role only at higher temperatures due to the thermal activation of free carriers (holes) into the valence band. Interestingly, the conductivity ratio along the two main crystallographic directions of the material, the key parameter defining the anisotropy of the system, varies nontrivially with respect to both temperature and 
carrier density. By using a simple model, we demonstrate that this behavior is due to the coexistence of VRH and band conduction in the system.

\section{EXPERIMENT METHOD}

Layered GeAs crystalizes in the monoclinic structure in the space group $C / 2 m\left(C_{2 h}{ }^{3}\right)$ as shown in Fig. 1(a) [22]. In order to measure the resistivity along the main crystallographic armchair and zigzag directions ( $\rho_{\mathrm{ac}}$ and $\rho_{\mathrm{zz}}$ ), we fabricated FET devices that are deliberately oriented by an angle $\theta$ relative to the crystallographic axes. The optical image of a typical device with a rotation angle of $51^{\circ}$ is shown in Fig. 1(b). In this configuration, the nonzero off-diagonal component in the rotated resistivity tensor [23]

$$
\begin{aligned}
& \left(\begin{array}{ll}
\rho_{x x} & \rho_{x y} \\
\rho_{y x} & \rho_{y y}
\end{array}\right) \\
& \quad=\left[\begin{array}{ll}
\rho_{\mathrm{ac}} \cos ^{2}(\theta)+\rho_{\mathrm{zz}} \sin ^{2}(\theta) & \left(\rho_{\mathrm{ac}}-\rho_{\mathrm{zz}}\right) \cos (\theta) \sin (\theta) \\
\left(\rho_{\mathrm{ac}}-\rho_{\mathrm{zz}}\right) \cos (\theta) \sin (\theta) & \rho_{\mathrm{zz}} \cos ^{2}(\theta)+\rho_{\mathrm{ac}} \sin ^{2}(\theta)
\end{array}\right]
\end{aligned}
$$

gives rise to a transverse voltage $V_{x y}$ when a source-drain current $I_{S D}$ (or source-drain voltage $V_{S D}$ ) is applied along the $x$ direction. Thus, the longitudinal and transverse resistivity $\left(\rho_{x x}\right.$ and $\left.\rho_{x y}\right)$ can be determined by measuring the transverse voltage $V_{x y}$ and the longitudinal one $V_{x x}$. Consequently, $\rho_{\mathrm{ac}}$ and $\rho_{\mathrm{zz}}$ can be simultaneously extracted by using the rotated resistivity tensor. This method has been applied to measure the anisotropic electrical resistivity in other 2D systems where anisotropy is given by charge-density-wave (CDW) ordering [23] or superimposed periodic potential modulation [24,25]. The devices are fabricated using GeAs flakes of approximately $12 \mathrm{~nm}$ thickness that are exfoliated on degenerately $p$-doped silicon wafers with a 300-nm oxide layer on top. The rotation angles of the devices are determined using angle-resolved polarized Raman spectroscopy. More details about device fabrication and determination of the rotation angle can be found in Appendix A and the Supplemental Material [26,27].

\section{RESULTS AND DISCUSSION}

The extracted conductivity along the armchair and zigzag directions $\left(\sigma_{\mathrm{ac}}\right.$ and $\left.\sigma_{\mathrm{zz}}\right)$ are plotted as a function of the applied gate voltage ( $\left.V_{g}\right)$ in Figs. 2(a) and 2(b), respectively. Three initial observations can be made. Firstly, the conductivity along the zigzag direction is higher than that along the armchair direction. Secondly, the sample shows typical $p$-type semiconductor behavior along both directions. Both of these observations are consistent with previous reports on FET devices made of this material $[10,11]$. Lastly, the modulation of the conductivity by $V_{g}$ is
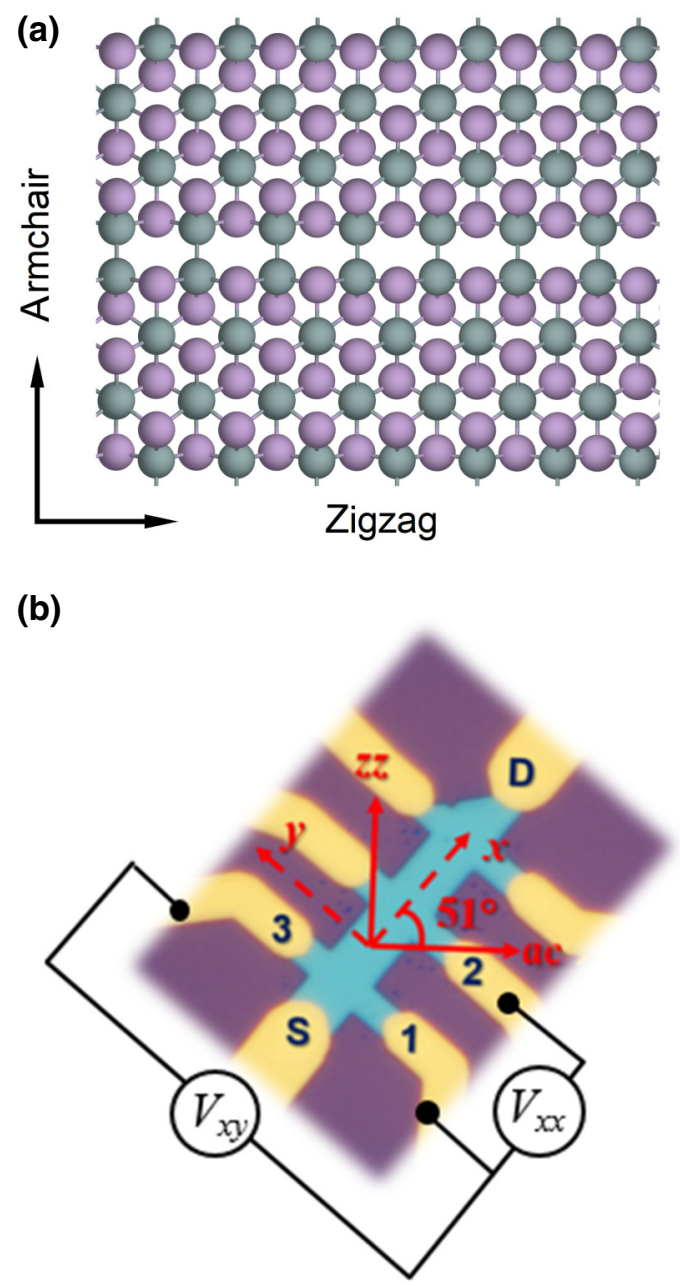

FIG. 1. (a) In-plane crystal structure of GeAs (Ge, green spheres; As, purple spheres). (b) Optical image of a device with rotation angle of $51^{\circ}$ and the electrical connection for measuring $V_{x x}$ and $V_{x y}$.

very weak (less than 1 order of magnitude) and the channel cannot be turned off within the applied gate-voltage range $(-60 \mathrm{~V}$ to $+60 \mathrm{~V})$. Moreover, from the data in Figs. 2(a) and 2(b), we notice that the conductivity is not linear with respect to $V_{g}$ in the range from $-60 \mathrm{~V}$ to $+60 \mathrm{~V}$; rather, the conductivity follows a power dependence on the carrier density $n$ :

$$
\sigma \propto n^{\alpha}
$$

According to theory $[28,29], \alpha$ is expected to be (i) $<1$ if short-range potential dominates the scattering of the carrier transport, and (ii) $\gtrsim 1$ if Coulomb scattering dominates. In our case, by fitting the conductivity plots in Figs. 2(a) and 2(b) at all the measured temperatures with Eq. (2), we extract $1.2 \leq \alpha \leq 2$. These values indicate that carrier transport in our devices is dominated by Coulomb scattering introduced by the charged impurities in the system. 


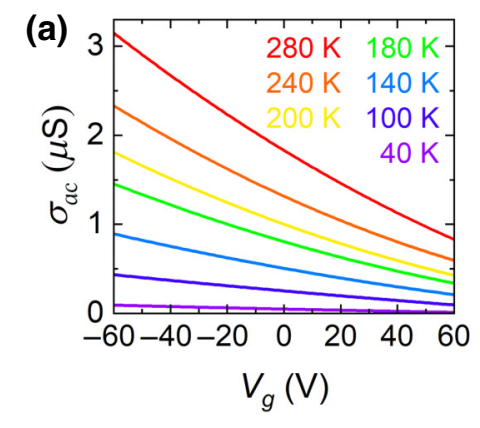

(c)

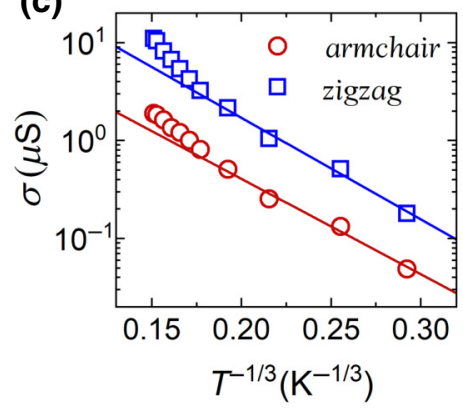

(d)

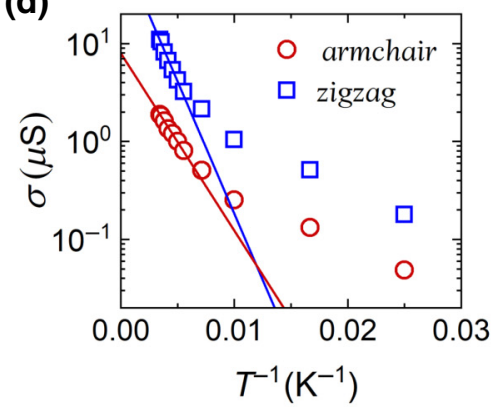

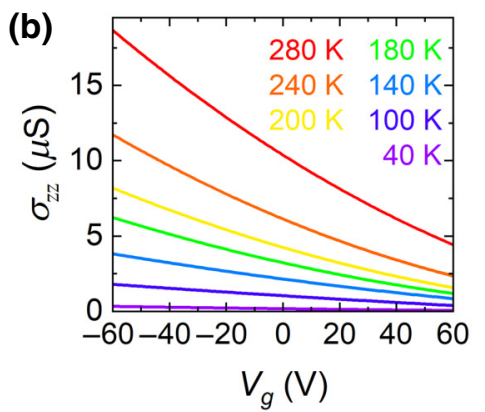

(e)

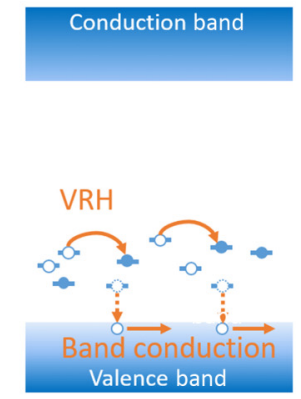

FIG. 2. Conductivity along the (a) armchair and (b) zigzag directions at different temperatures as a function of the gate voltage. Conductivity along both crystallographic directions as a function of (c) $T^{-1 / 3}$ and (d) $T^{-1}$. (e) Schematic illustration of the VRH via localized states in the band gap and the thermal activation of the free carriers (holes) into the valence band in disordered systems.

A similar observation was reported in a previous experimental study on $\mathrm{MoS}_{2}$ [30]. Furthermore, we notice that $\alpha$ shows a strong dependence on temperature, in agreement with theoretical predictions that in 2D systems the screening of the Coulomb scattering is temperature dependent in a nontrivial way [29,31].

The temperature dependence of the conductivity at a constant gate voltage further emphasizes the cause for the observed gate dependence of the conductivity. As shown in Fig. 2(c) for $V_{g}=0 \mathrm{~V}$, at low temperatures $(T<180 \mathrm{~K})$, $\sigma_{\mathrm{ac}}$ and $\sigma_{\mathrm{zz}}$ follow a linear dependence on $T^{-1 / 3}$. This is a clear signature of VRH transport via localized states as described by the 2D Mott formalism [28]

$$
\sigma \sim \exp \left[-\left(\frac{T_{0}}{T}\right)^{\frac{1}{3}}\right]
$$

in which $T_{0}$ corresponds to the correlation energy scale [32]. The fact that the data can be well fitted with the 2D Mott formalism is a further proof that our material effectively behaves as a $2 \mathrm{D}$ conductor. VRH transport normally occurs in polycrystalline crystals [32] and organic materials [33], but has also been observed in many other 2D materials with a notable density of defects, such as $\mathrm{MoS}_{2}$ [30,34], black phosphorus [35], and other groupIV-V compound 2D materials [22]. The observation of VRH confirms the presence of impurities in the studied system, which is nonetheless expected for $2 \mathrm{D}$ materials, which, like GeAs, are not very stable in air [10,36]. Nevertheless, other effects, for instance vacancies or the presence of trapped states at the interface with substrate, could also play a role.

At higher temperature $(T>180 \mathrm{~K}), \sigma_{\mathrm{ac}}$ and $\sigma_{\mathrm{zz}}$ instead show an exponential decrease proportional to $T^{-1}$ as shown in Fig. 2(d). This indicates the thermal activation of carriers (holes) from localized states in the band gap to the valence band in our devices, leading to a band-conduction regime described by the expression [37]

$$
\sigma=\sigma_{0} \exp \left(-\frac{E_{a}}{k_{B} T}\right)
$$

where $E_{a}$ is the activation energy, $k_{B}$ is the Boltzmann constant, and $\sigma_{0}$ is a fitting parameter. Figure 2(e) illustrates schematically both conduction mechanisms observed in these samples, namely VRH via localized states and band conduction due to the thermal activation of the free carriers to the valence band.

Next, we focus our attention on the ratio $\left(\sigma_{\mathrm{zz}} / \sigma_{\mathrm{ac}}\right)$ of conductivities along zigzag and armchair directions. This ratio is the key performance indicator of the electrical conduction in anisotropic materials. It provides information on the effective anisotropic transport achieved in real devices, accounting for both the intrinsic anisotropy conduction expected due to the crystal structure, and the conduction due to the presence of impurities. To a first approximation, electrical transport in semiconductors with band-edge conduction takes place exclusively around 
(a)

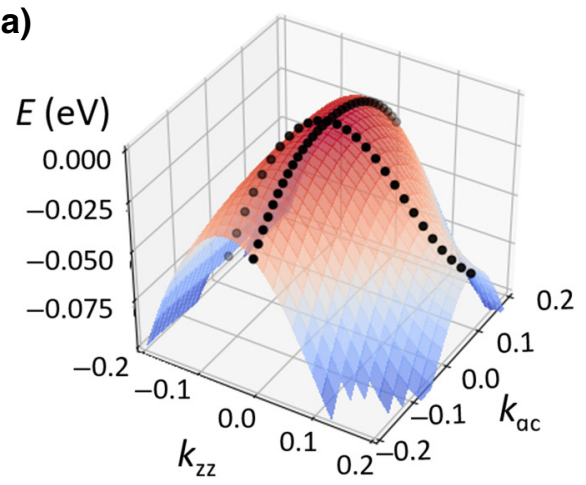

(c)

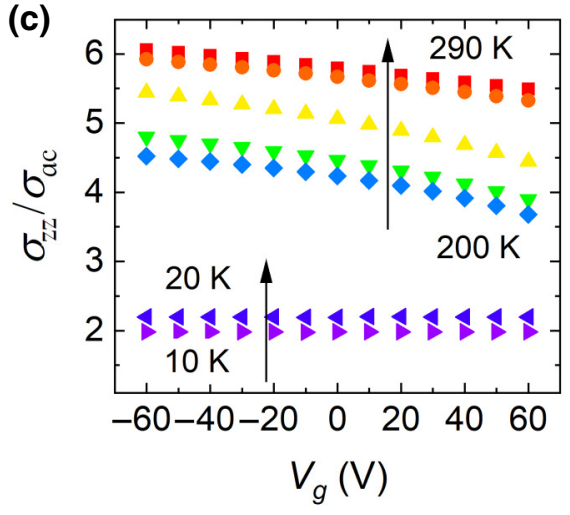

(b)

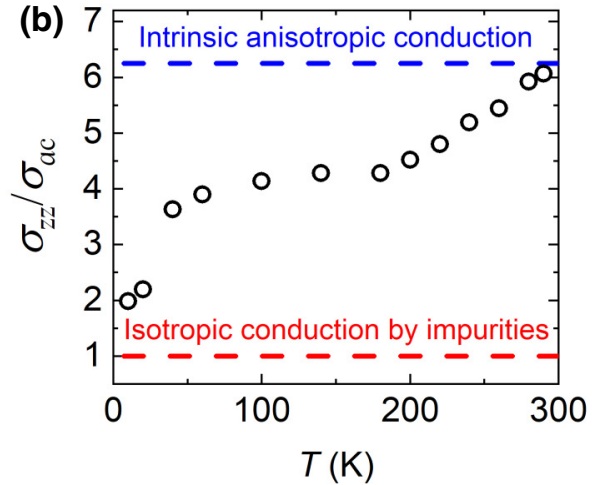

(d)

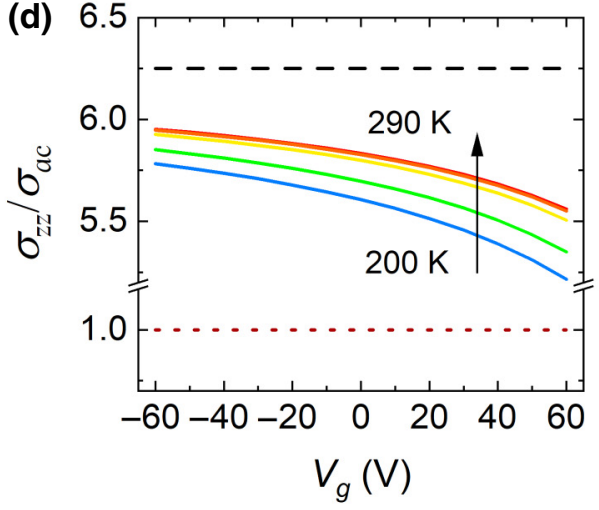

FIG. 3. (a) 3D plot of the energy dispersion at VBM along the armchair and zigzag directions. Conductivity ratio as a function of (b) temperature and (c) $V_{g}$. (d) Simulation of the conductivity ratio as a function of $V_{g}$. the conduction-band minimum (CBM for $n$-type semiconductors) or valence-band maximum (VBM for $p$-type semiconductors). Thus, according to Drude's model [38]

$$
\sigma=\frac{e^{2} \tau n^{\text {band }}}{m^{*}}
$$

and assuming a relaxation time of free carriers $\tau$ and the carrier density in band conduction $n^{\text {band }}$ independent of the lattice direction, the conductivity ratio in intrinsic anisotropic materials should be constant and given by the ratio of the effective masses $m^{*}$ along the two crystallographic directions (here the effective masses of holes along the armchair and zigzag directions, $m_{\mathrm{ac}}^{h *}$ and $m_{\mathrm{zz}}^{h *}$, respectively). We estimate this anisotropic conductivity ratio in GeAs by means of first-principles density-functionaltheory (DFT) simulations. First, we calculate the band structure of bulk GeAs along the zigzag and armchair directions of the monoclinic structure. In Fig. 3(a) we show a $3 \mathrm{D}$ contour plot of the dispersion relation $E(k)$ around the VBM. Here, a larger dispersion is clearly visible along the zigzag with respect to the armchair direction. The calculated effective mass of holes at the VBM is $1.5 m_{o}$ ( $m_{o}$ being the electron rest mass) and $0.24 m_{o}$ along the armchair and zigzag directions, respectively, which gives a theoretical estimation of the conductivity ratio $\sigma_{\mathrm{zz}} / \sigma_{\mathrm{ac}}$ of 6.25 . We note that this value is close to the one found in previous calculations [11]. Details about the atomic structure, band structure, and effective-mass calculation are given within the Supplemental Material [39-44].

Interestingly, our experimentally measured $\sigma_{\mathrm{zz}} / \sigma_{\mathrm{ac}}$ ratio is, however, not constant and exhibits an intriguing dependence on both temperature and gate voltage. As shown in Fig. 3(b), at the highest temperature $(290 \mathrm{~K}), \sigma_{\mathrm{zz}} / \sigma_{\mathrm{ac}}$ is found to be around 6 , but as temperature decreases, so does $\sigma_{\mathrm{zz}} / \sigma_{\mathrm{ac}}$ and tends to unity when temperature approaches $0 \mathrm{~K}$.

The small $\sigma_{\mathrm{zz}} / \sigma_{\mathrm{ac}}$ ratio measured at low temperature is consistent with our previous observation of isotropic VRH being the dominant transport mechanism at low temperatures, as shown in Fig. 2(c). Indeed, considering a random distribution of the impurities, it is reasonable that isotropic VRH occurs in our devices. As temperature increases, band conduction in the material progressively becomes more dominant in comparison with VRH, which explains the measured increment of the conductivity ratio (towards the intrinsic value). Nevertheless, we emphasize that even at room temperature, i.e., at application-relevant conditions, the measured $\sigma_{\mathrm{zz}} / \sigma_{\mathrm{ac}}$ is still smaller than the theoretically predicted effective-mass ratio (6.25), suggesting that VRH still plays a role in the carrier transport. Moreover, the observed modulation of $\sigma_{\mathrm{zz}} / \sigma_{\mathrm{ac}}$ with respect to the gate voltage at different temperatures [Fig. 3(c)] is also consistent with VRH and band-conduction transport mechanisms coexisting simultaneously in the system; at lower $V_{g}$, more free carriers are injected into the valence band, 
which eventually leads to a higher band conduction and subsequently higher conductivity ratio.

We underpin all these observations by developing a model, which, accounting for the interplay between both band conduction and VRH transport mechanisms, is able to qualitatively explain the measured evolution of the conductivity ratio as a function of $T$ and $V_{g}$. In general terms, the conductivity can be described as the sum [45] of the hopping conductivity $\left(\sigma^{\mathrm{VRH}}\right)$ and the band conductivity [46] ( $\left.\sigma^{\text {band }}\right)$ as given by the expression:

$$
\sigma\left(V_{g}\right)=\sigma^{\text {band }}+\sigma^{\mathrm{VRH}}=\mu C_{g}\left|V_{g}-V_{\mathrm{th}}\right|+\sigma^{\mathrm{VRH}},
$$

where $V_{\text {th }}$ is the voltage needed to compensate the doping introduced by impurities with the gate, $\mu$ is the mobility of the carriers, and $C_{g}$ is the gate capacitance per unit area. Due to the assumed random distribution of impurities in our system, we impose $\sigma^{\mathrm{VRH}}$ to be constant along zigzag and armchair directions, whereas $\mu$ is different $\left(\mu_{\mathrm{zz}}\right.$ and $\mu_{\mathrm{ac}}$ being the carrier mobility along the zigzag and armchair directions, respectively). Thus, the overall conductivity ratio $\left(\sigma_{\mathrm{zz}} / \sigma_{\mathrm{ac}}\right)$ in the system can be written as

$$
\frac{\sigma_{\mathrm{zz}}}{\sigma_{\mathrm{ac}}}=\frac{e n^{\mathrm{band}} \mu_{\mathrm{zz}}^{\mathrm{band}}+\sigma^{\mathrm{VRH}}}{e n^{\mathrm{band}} \mu_{\mathrm{ac}}^{\mathrm{band}}+\sigma^{\mathrm{VRH}}} .
$$

As we can see, this model [Eq. (7)] reflects well the two aforementioned limit scenarios. Assuming $\sigma^{\mathrm{VRH}} \gg \sigma^{\text {band }}$, carrier transport is dominated by VRH and $\sigma_{\mathrm{zz}} / \sigma_{\mathrm{ac}}$ is 1 , independent of the gate voltage [dotted lines in Fig. 3(d)]. On the other hand, assuming $\sigma^{\mathrm{VRH}} \ll \sigma^{\text {band }}, \frac{\sigma_{\mathrm{ZZ}}}{\sigma_{\mathrm{ac}}} \sim \frac{\mu_{\mathrm{ZZ}}^{\text {band }}}{\mu_{\mathrm{ac}}^{\text {band }}}=$ 6.25 at any gate voltage [dashed lines in Fig. 3(d)], i.e., the electrical conduction is anisotropic as a result of the intrinsic crystal structure of the material, with $\sigma_{\mathrm{zz}} / \sigma_{\mathrm{ac}}$ given by the ratio of effective masses along zigzag and armchair directions. When $\sigma^{\mathrm{VRH}}$ is comparable to $\sigma_{\mathrm{ac}}^{\text {band }}$ and $\sigma_{\mathrm{zz}}^{\text {band }}$, an intermediate behavior in which $\sigma_{\mathrm{zz}} / \sigma_{\mathrm{ac}}$ depends on the gate voltage is expected. This is indeed shown in Fig. 3(d), where we calculate without fitting parameters the conductivity ratio as a function of $V_{g}$. The overall calculation is in remarkable qualitative agreement with the experimentally observed $V_{g}$ dependence of the conductivity ratio [Fig. 3(c)]. Specifically, as $V_{g}$ decreases towards $-60 \mathrm{~V}$, more free carriers are injected into the valence band, which makes the contribution of $\sigma^{\text {band }}$ larger than $\sigma^{\mathrm{VRH}}$, which leads to an increasing of the conductivity ratio. Moreover, our model can well account for the temperature dependence of the conductivity ratio and its modulation by the gate voltage. Firstly, as shown in Figs. 3(b) and 3(c), a decrease in conductivity ratio is observed for lower temperatures, which is due to the fact that at lower temperature $\sigma^{\text {band }}$ contributes less than $\sigma^{\mathrm{VRH}}$ to the overall conductance, hence the conductivity ratio tends towards unity (the case of pure VRH). At low temperatures (e.g., 10
TABLE I. Contributions of VRH and band conduction to the overall conductivity at three critical temperatures $(10,180$, and $290 \mathrm{~K})$ at $V_{g}=-60 \mathrm{~V}$.

\begin{tabular}{lcccccc}
\hline \hline & & \multicolumn{2}{c}{ Zigzag } & & \multicolumn{2}{c}{ Armchair } \\
\cline { 3 - 4 } \cline { 6 - 7 }$T$ & $\Delta\left(\sigma_{\mathrm{zz}} / \sigma_{\mathrm{ac}}\right)^{\mathrm{a}}$ & $\mathrm{VRH}$ & $\begin{array}{c}\text { Band } \\
\text { conduction }\end{array}$ & & VRH & $\begin{array}{c}\text { Band } \\
\text { conduction }\end{array}$ \\
\hline $10 \mathrm{~K}$ & 0 & $40 \%$ & $60 \%$ & & $81 \%$ & $19 \%$ \\
$200 \mathrm{~K}$ & 0.84 & $7 \%$ & $93 \%$ & & $30 \%$ & $70 \%$ \\
$290 \mathrm{~K}$ & 0.57 & $1 \%$ & $99 \%$ & & $5 \%$ & $95 \%$ \\
\hline \hline
\end{tabular}

${ }^{\text {a }}$ Represents the difference between the conductivity ratio measured at $V_{g}=-60 \mathrm{~V}$ and that one measured $V_{g}=60 \mathrm{~V}$.

and $20 \mathrm{~K}$ ), the conductivity ratio exhibits much weaker modulation by $V_{g}$ than those at intermediate temperatures $(140-220 \mathrm{~K})$. This is clearly reflected by our model, that is, at lower temperature, VRH makes a larger contribution to the overall conductivity than band conduction; as a result, the conductivity ratio exhibits much weaker modulation by $V_{g}$ since one conduction mechanism clearly dominates over the other. Secondly, around $200 \mathrm{~K}$, the variation of the conductivity ratio for the measured $V_{g}$ range is the strongest. This is because here VRH and band conduction have a comparable contribution to the overall conductivity. Finally, as temperature increases further, the modulation of the conductivity ratio decreases, because band conduction dominates the overall conductivity in this range. In Table I, we list the contributions of VRH and band conduction to the overall conductivity at three critical temperatures $(10$, 180 , and $290 \mathrm{~K}$ ), which correspond to the changes of the conductivity ratio as shown in Fig. 3(b). Additionally, we note that the maximum conductivity ratio measured in our devices (approximately 6 at $T=290 \mathrm{~K}$ and $V_{g}=-60 \mathrm{~V}$ ) approaches the theoretically predicted effective-mass ratio (6.25), which is also in agreement with our simple model. Details regarding the model and further comparison with experimental values are reported in Appendix B.

\section{CONCLUSION}

In summary, we experimentally study the electronic transport in transistor devices made from the anisotropic layered model system GeAs. Notably, we first measure the conductivity along the two relevant crystallographic directions by means of the transverse voltage method in the temperature range from room temperature down to $10 \mathrm{~K}$. Then, we show that the extracted conductivity ratio is close to the theoretically predicted value (6.25) at room temperature and high carrier densities $\left(V_{g}=-60 \mathrm{~V}\right)$. However, rather than being constant, it exhibits a peculiar dependence with gate and temperature. This dependence is explained by considering the coexistence of both the intrinsic anisotropic band conduction and the isotropic VRH induced by random-distributed impurities in the material. 
As impurities, such as structural defects or surface contamination, are in general extremely hard to avoid in devices made of 2D materials, we expect that our results will be beneficial for the exploitation of these materials in practical applications.

\section{ACKNOWLEDGMENTS}

This research is supported by the Villum Fonden through the Young Investigator Program (Project No. 19130). J.M.C. acknowledges financial support from the Danish National Research Foundation Center for Nanostructured Graphene Project No. DNRF103. M.P. is grateful to INFN for financial support through the National project Nemesys and ISCRA-B initiative for granting access to computational resources on Marconi at CINECA, Italy. L.C. acknowledges support from the Italian Ministry of Education, University and Research (MIUR) via "Programma per Giovani Ricercatori - Rita Levi Montalcini 2017”.

\section{APPENDIX A}

Devices are fabricated using GeAs thin flakes (2D semiconductors) that are mechanically exfoliated onto Si wafer with a $300-n m$ oxide layer. The FET structures are shaped using electron-beam lithography using a LEO scanning electron microscope (SEM) integrated with a Raith Elphy System, followed by dry etching with sulfur hexafluoride $\left(\mathrm{SF}_{6}\right)$ using a STS inductively coupled plasma system. The electrodes are fabricated using a standard lift-off process with nickel $(20 \mathrm{~nm})$ and gold $(50 \mathrm{~nm})$ that are deposited by electron-beam evaporation using a Temescal E-beam evaporator. The electrical measurements are performed in an Oxford Instrument Teslatron PT cryostat. After the electrical measurement, the crystal orientation of the material is determined based on the angle-resolved polarized Raman spectrum measured using a Labram Micro Raman apparatus by Jobin Yvon with approximately $0.3 \mathrm{~mW}$, $\lambda_{0}=632 \mathrm{~nm}$ excitation in backscattering geometry through a $100 \times$ long working distance objective.

\section{APPENDIX B}

In our model, we keep $\mu_{\mathrm{zz}}^{\mathrm{band}} / \mu_{\mathrm{ac}}^{\mathrm{band}}$ constant as the theoretically value of the effective-mass ratio, $6.25 . \mu_{\mathrm{ac}}^{\mathrm{band}}$ is taken from the experimental value at $V_{g}=-60 \mathrm{~V}$, which is considered to be the best approximation to the mobility of the free carriers (in band conduction) at the different temperatures. We take the residual carrier density of the material at null gate voltage (i.e., doping induced by impurities) $n_{0}=C_{g} V_{\text {th }}$, with $V_{\text {th }}$ being the voltage needed to compensate the doping introduced by impurities. In our devices, $V_{\text {th }} \sim 140 \mathrm{~V}$ is extracted by extrapolating the $\sigma$ versus $V_{g}$ curve at $290 \mathrm{~K}$ based on the quadratic fitting. Finally, $\sigma^{\mathrm{VRH}}$ is taken as the conductivity at the VRH to band-conduction transition temperature $(180 \mathrm{~K})$ at $V_{g}=$ $60 \mathrm{~V}$, experimental conditions with a minimal amount of free carriers induced by gate voltage and temperature.

As shown in Figs. 3(c) and 3(d), despite the simple model used, the simulation results are qualitatively in good agreement with the experimental results. At $290 \mathrm{~K}$, the simulation is in quantitative agreement with experimental data. However, the calculated conductivity ratios show values higher than the experimentally measured ones at lower temperature (for instance at $T=200 \mathrm{~K}$, the calculated $\sigma_{\mathrm{zz}} / \sigma_{\mathrm{ac}}$ at $V_{g}=-60 \mathrm{~V}$ is approximately 5.7 ; whereas the measured value is approximately 4.5). This disagreement, however, can be easily explained by the fact that the bandconduction parameters used for the simulations are taken from the overall experimental conductivity, which however consists of contributions from both VRH and band conduction (i.e., it is not exclusively due to band conduction). Thus, the contribution of band conduction included in our model is overestimated at those low temperatures $(200-280 \mathrm{~K})$. Nevertheless, we emphasize that in its simplicity our model is still able to capture qualitatively the observed behavior.

[1] L. Li, W. Han, L. Pi, P. Niu, J. Han, C. Wang, B. Su, H. Li, J. Xiong, Y. Bando, and T. Zhai, Emerging inplane anisotropic two-dimensional materials, InfoMat 1, 54 (2019).

[2] E. Liu, Y. Fu, Y. Wang, Y. Feng, H. Liu, X. Wan, W. Zhou, B. Wang, L. Shao, C.-H. Ho et al., Integrated digital inverters based on two-dimensional anisotropic $\mathrm{ReS}_{2}$ field-effect transistors, Nat. Commun. 6, 6991 (2015).

[3] Y. Liu and K.-W. Ang, Monolithically integrated flexible black phosphorus complementary inverter circuits, ACS Nano 11, 7416 (2017).

[4] H. Yuan, X. Liu, F. Afshinmanesh, W. Li, G. Xu, J. Sun, B. Lian, A. G. Curto, G. Ye, Y. Hikita et al., Polarizationsensitive broadband photodetector using a black phosphorus vertical p-n junction, Nat. Nanotechnol. 10, 707 (2015).

[5] L. Li, P. Gong, D. Sheng, S. Wang, W. Wang, X. Zhu, X. Shi, F. Wang, W. Han, S. Yang, K. Liu, H. Li, and T. Zhai, Highly in-plane anisotropic 2D $\mathrm{GeAs}_{2}$ for polarizationsensitive photodetection, Adv. Mater. 30, 1804541 (2018).

[6] F. Xia, H. Wang, and Y. Jia, Rediscovering black phosphorus as an anisotropic layered material for optoelectronics and electronics, Nat. Commun. 5, 4458 (2014).

[7] D. Tan, H. E. Lim, F. Wang, N. B. Mohamed, S. Mouri, W. Zhang, Y. Miyauchi, M. Ohfuchi, and K. Matsuda, Anisotropic optical and electronic properties of twodimensional layered germanium sulfide, Nano Res. 10, 546 (2017).

[8] Y. C. Lin, H. P. Komsa, C. H. Yeh, T. Björkman, Z. Y. Liang, C. H. Ho, Y. S. Huang, P. W. Chiu, A. V. Krasheninnikov, and K. Suenaga, Single-layer $\operatorname{ReS}_{2}$ : Two-dimensional semiconductor with tunable in-plane anisotropy, ACS Nano 9, 11249 (2015). 
[9] F. Liu, H. Shimotani, H. Shang, T. Kanagasekaran, V. Zólyomi, N. Drummond, V. I. Fal'ko, and K. Tanigaki, High-sensitivity photodetectors based on multilayer GaTe flakes, ACS Nano 8, 752 (2014).

[10] J. Guo, Y. Liu, Y. Ma, E. Zhu, S.-J. J. S. Lee, Z. Lu, Z. Zhao, C. Xu, H. Wu, K. Kovnir, Y. Huang, and $X$. Duan, Few-layer GeAs field-effect transistors and infrared photodetectors, Adv. Mater. 30, 1705934 (2018).

[11] S. Yang, Y. Yang, M. Wu, C. Hu, W. Shen, Y. Gong, L. Huang, C. Jiang, Y. Zhang, and P. M. Ajayan, Highly inplane optical and electrical anisotropy of 2D germanium arsenide, Adv. Funct. Mater. 28, 1707379 (2018).

[12] L. Li, W. Wang, P. Gong, X. Zhu, B. Deng, X. Shi, G. Gao, H. Li, and T. Zhai, 2D gep: An unexploited low-symmetry semiconductor with strong in-plane anisotropy, Adv. Mater. 30, 1706771 (2018).

[13] S. Ihnatsenka, Activated hopping transport in anisotropic systems at low temperatures, Phys. Rev. B 94, 195202 (2016).

[14] V. K. Sangwan and M. C. Hersam, Electronic transport in two-dimensional materials, Annu. Rev. Phys. Chem. 69, 299 (2018).

[15] Z. Tian, C. Guo, M. Zhao, R. Li, and J. Xue, Twodimensional SnS: A phosphorene analogue with strong in-plane electronic anisotropy, ACS Nano 11, 2219 (2017).

[16] Y. Chen, C. Chen, R. Kealhofer, H. Liu, Z. Yuan, L. Jiang, J. Suh, J. Park, C. Ko, H. S. Choe, et al., Black arsenic: A layered semiconductor with extreme in-plane anisotropy, Adv. Mater. 30, 1800754 (2018).

[17] G. Qiu, Y. Du, A. Charnas, H. Zhou, S. Jin, Z. Luo, D. Y. Zemlyanov, X. Xu, G. J. Cheng, and P. D. Ye, Observation of optical and electrical in-plane anisotropy in high-mobility few-layer $\mathrm{ZrTe}_{5}$, Nano Lett. 16, 7364 (2016).

[18] K. Lee, S. Kamali, T. Ericsson, M. Bellard, and K. Kovnir, Geas: Highly anisotropic van der waals thermoelectric material, Chem. Mater. 28, 2776 (2016).

[19] C. R. Kannewurf and R. J. Cashman, Optical absorption and photoconductivity in germanium selenide, J. Phys. Chem. Solids 22, 293 (1961).

[20] L. Zhou, Y. Guo, and J. Zhao, GeAs and SiAs monolayers: Novel 2D semiconductors with suitable band structures, Phys. E Low-Dimensional Syst. Nanostructures 95, 149 (2018).

[21] B. Mortazavi and T. Rabczuk, Anisotropic mechanical properties and strain tunable band-gap in single-layer SiP, SiAs, GeP and GeAs, Phys. E Low-Dimens. Syst. Nanostructures 103, 273 (2018).

[22] C. Barreteau, B. Michon, C. Besnard, and E. Giannini, High-pressure melt growth and transport properties of SiP, SiAs, GeP, and GeAs 2D layered semiconductors, J. Cryst. Growth 443, 75 (2016).

[23] P. Walmsley and I. R. Fisher, Determination of the resistivity anisotropy of orthorhombic materials via transverse resistivity measurements, Rev. Sci. Instrum. 88, 043901 (2017).

[24] J. M. Caridad, S. Connaughton, C. Ott, H. B. Weber, and V. Krstić, An electrical analogy to Mie scattering, Nat. Commun. 7, 12894 (2016).
[25] T. Schlösser, K. Ensslin, F. Claro, J. P. Kotthaus, M. Holland, and R. Ketzmerick, Corrugation-induced transverse voltage in a lateral superlattice, Phys. Rev. B 51, 10737 (1995).

[26] See Supplemental Material at http://link.aps.org/supplemen tal/10.1103/PhysRevApplied.13.044063 for details about the determination of the crystal orientation.

[27] R. Loudon, The Raman effect in crystals, Adv. Phys. 13, 423 (1964).

[28] S. Adam and S. Das Sarma, Boltzmann transport and residual conductivity in bilayer graphene, Phys. Rev. B 77, 115436 (2008).

[29] K. Kaasbjerg, T. Low, and A.-P. Jauho, Electron and hole transport in disordered monolayer $\mathrm{MoS}_{2}$ : Atomic vacancy induced short-range and Coulomb disorder scattering, Phys. Rev. B 100, 115409 (2019).

[30] S. Ghatak, A. N. Pal, and A. Ghosh, Nature of electronic states in atomically thin $\mathrm{MoS}_{2}$ field-effect transistors, ACS Nano 5, 7707 (2011).

[31] S. Das Sarma and E. H. Hwang, Screening and transport in 2D semiconductor systems at low temperatures, Sci. Rep. 5, 16655 (2015).

[32] N. F. Mott and E. A. Davis, Electronic Processes in NonCrystalline Materials (Oxford University Press, New York, 1971).

[33] P. Stallinga, Electronic transport in organic materials: Comparison of band theory with percolation/(variable range) hopping theory, Adv. Mater. 23, 3356 (2011).

[34] W. Zhu, T. Low, Y. H. Lee, H. Wang, D. B. Farmer, J. Kong, F. Xia, and P. Avouris, Electronic transport and device prospects of monolayer molybdenum disulphide grown by chemical vapour deposition, Nat. Commun. 5, 3087 (2014).

[35] S. Jae Choi, B.-K. Kim, T.-H. Lee, Y. Ho Kim, Z. Li, E. Pop, J.-J. Kim, J. Hyun Song, and M.-H. Bae, Electrical and thermoelectric transport by variable range hopping in thin black phosphorus devices, Nano Lett. 16, 3969 (2016).

[36] J. Sun, G. Giorgi, M. Palummo, P. Sutter, M. Passacantando, and L. Camilli, A scalable method for thickness and lateral engineering of 2D materials, ACS Nano. (2020).

[37] D. A. Neamen, Semiconductor Physics and DevicesâĂŕ: Basic Principles (McGraw-Hill, New York, 2011).

[38] P. Drude, Zur elektronentheorie der metalle, Ann. Phys. 306, 566 (1900).

[39] See Supplemental Material at http://link.aps.org/supplemen tal/10.1103/PhysRevApplied.13.044063 for details about calculation of the effective-mass ratio.

[40] P. Giannozzi, S. Baroni, N. Bonini, M. Calandra, R. Car, C. Cavazzoni, D. Ceresoli, G. L. Chiarotti, M. Cococcioni, I. Dabo, et al., Quantum ESPRESSO: A modular and open-source software project for quantum simulations of materials, J. Phys. Condens. Matter 21, 395502 (2009).

[41] J. P. Perdew, A. Ruzsinszky, G. I. Csonka, O. A. Vydrov, G. E. Scuseria, L. A. Constantin, X. Zhou, and K. Burke, Restoring the Density-Gradient Expansion for Exchange in Solids and Surfaces, Phys. Rev. Lett. 100, 136406 (2008).

[42] S. Grimme, Semiempirical GGA-type density functional constructed with a long-range dispersion correction, J. Comput. Chem. 27, 1787 (2006). 
[43] M. Schlipf and F. Gygi, Optimization algorithm for the generation of ONCV pseudopotentials, Comput. Phys. Commun. 196, 36 (2015).

[44] B. Van Zeghbroeck, Principles of Semiconductor Devices and Heterojunctions, 1st ed (Prentice Hall, London, 2007).
[45] T. Chen and B. Skinner, Enhancement of hopping conductivity by spontaneous fractal ordering of low-energy sites, Phys. Rev. B 94, 85146 (2016).

[46] J.-H. H. Chen, C. Jang, S. Adam, M. S. Fuhrer, E. D. Williams, and M. Ishigami, Charged-impurity scattering in graphene, Nat. Phys. 4, 377 (2008). 